Territorios 32 / Bogotá, 2015, pp. 61-79

ISSN: 0123-8418

ISSNe: 2215-7484

Procesos de ocupación del territorio, historia urbana y patrimonio (I)

\title{
Del patrimonio a la cultura: evoluciones en la gobernanza urbana de Quito
}

From Heritage to Culture: Evolutions in Urban Governance in Quito

Do património à cultura: evoluções na governança urbana de Quito

Diana Burgos-Vigna*

Recibido: 15 de septiembre de 2014

Aprobado: 6 de noviembre de 2014

Doi: dx.doi.org/10.12804/territ32.2015.03

Para citar este artículo:

Burgos-Vigna, D. (2015). Del patrimonio a la cultura: evoluciones en la gobernanza urbana de Quito. Territorios, 32, 61-79. Doi: dx.doi.org/10.12804/territ32.2015.03

* Licenciada en Ciencias Politicas de la Universidad de Aix-Marseille, $y$ tesis doctoral en civilización latinoamericana, en la Universidad Sorbonne Nouvelle (Paris 3) en 2003. Profesora titular de la Universidad de CergyPontoise (Francia). Equipo de investigación AGORA. Correo electrónico : Diana. burgos@u-cergy.fr 
Palabras clave

Gobernanza urbana, centro histórico, politica cultural, patrimonio.

Keywords

Urban governance, historical, cultural policy, heritage.

Palavras-chave Governança urbana, centro histórico, politica cultural, património.

\section{territarias 32}

\section{RESUMEN}

Quito se convierte en 1978 en la primera ciudad, con Cracovia, en recibir por parte de la Unesco la distinción de Patrimonio Cultural de la Humanidad. El título, otorgado a la ciudad por la riqueza de su centro histórico, permite crear un nuevo espacio de gobernanza urbana, que se analiza como un campo patrimonial relativamente autónomo. El estudio de los actores involucrados en este campo, que intervienen en diferentes escalas $-\mathrm{y}$ de las definiciones oficiales de patrimonio producidas en este campo-, desde los años ochenta, ayuda a identificar con mayor claridad las evoluciones actuales y ver en qué medida son innovadores. Por lo tanto, el análisis de dos programas implementados en los últimos años por el gobierno municipal - la campaña Cuéntame tu Quito (un proceso participativo sobre el concepto de patrimonio) y la construcción del Parque Urbano Qmandá (una infraestructura deportiva y cultural) - permite observar la emergencia de políticas ya no solo patrimoniales sino culturales. Integradas en un marco ideológico nacional definido desde 2008 por la Constitución de Montecristi, el del Estado del Buen Vivir, reflejan el surgimiento de una nueva relación entre los quiteños y su patrimonio y, más allá, entre los ciudadanos y su ciudad.

\section{ABSTRACT}

In 1978, Quito became the first city (with Cracovia) to be awarded the title of "Cultural Heritage of Humanity". The title, awarded by Unesco, for the cultural importance of the historic centre of the city, has helped to create a new understanding of urban governance, which leads us to the study of a "heritage field". The analysis of this field, and what has been happening on different scales for the city as well as the different official definitions given to the word "heritage" since the 1980s, allows us to identify how such a definition has evolved and what innovations it has led to. Thus, the study of a number of decisions taken in recent years by the municipal government in the field of heritage, like the municipal campaign Cuéntame tu Quito (a participatory process involving the concept of heritage) and the inauguration of the Parque Urbano Qmandá (a sports and cultural complex) have grown from simply heritage policies into real cultural policies. Since the Constitution of Montecristi in 2008, which has created the "Buen Vivir State", this change has been integrated into a national ideological framework, which allows us to see the emergence of a new relationship between the quiteño and its heritage and, beyond, between the citizen and his city.

\section{RESUMo}

Quito converte-se em 1978 na primeira cidade, com Cracóvia, em receber por parte da UNESCO a distinção de Património Cultural da Humanidade. O título, outorgado à cidade pela riqueza de seu centro histórico, permite criar um novo espaço de governança urbana, que analisamos como um "campo patrimonial” relativamente autónomo. O estudo dos atores envolvidos neste campo, que intervêm a diferentes escalas, e das definições oficiais de património produzidas neste campo, desde os anos 80, ajuda-nos a identificar com maior claridade as evoluções atuais e ver até que ponto são inovadoras. Pelo tanto, a análise de dois programas implementados nos últimos dois anos pelo governo municipal, a campanha Cuéntame tu Quito (um processo participativo sobre o conceito de património) e a construção do Parque Urbano Qmandá (uma infraestrutura desportiva e cultural) permite observar a emergência de políticas não só patrimoniais mas também culturais. Integradas 
em um marco ideológico nacional definido desde 2008 pela Constituição de Montecristi, o Estado de "Bom Viver", permite-nos ver o surgimento de uma nova relação entre os cidadãos de Quito e seu património, e mais além entre os cidadãos e sua cidade.

\section{Introducción}

En enero de 2014 se inauguró en Quito el parque urbano Qmandá, obra resultante de la rehabilitación de un antiguo terminal terrestre situado al sur del centro histórico de la ciudad. Este nuevo equipamiento fue presentado por las autoridades locales como:

El primer parque urbano que vincula las actividades deportivas con las culturales, fomentando diferentes escenarios y niveles de encuentro ciudadano que propician experiencias tendientes a plasmar a través de una gran obra pública los conceptos el Buen Vivir, en una ciudad contemporánea y cosmopolita como es el Quito de hoy (Fundación Museos, 2014, p. 69).

Así, se nota aquí la magnitud de un proyecto que supera los meros objetivos de planificación urbana y de revitalización del centro histórico para integrarse en un marco ideológico, ya no solo local sino nacional, que se refiere a la revolución ciudadana liderada por el presidente Rafael Correa.

Este objetivo es evidente también en la elección del Qmandá como escenario privilegiado para la exposición de los resultados de una gran consulta ciudadana llamada Cuéntame tu Quito. Esta consulta se organizó en 2013, con motivo del cumplimiento de los 35 años del nombra- miento del centro histórico de Quito como Patrimonio Cultural de la Humanidad por la Unesco. El objetivo oficial de la campaña fue, como se verá, ampliar y renovar la definición del patrimonio mediante la participación ciudadana, siendo también para el equipo municipal del entonces alcalde Augusto Barrera la oportunidad de dar más visibilidad a una política cultural innovadora llevada a cabo desde su elección en 2009.

Este trabajo de investigación ${ }^{1}$ se propone analizar el significado político y sociológico de estos dos proyectos (la construcción del Qmandá y la campaña Cuéntame tu Quito), ambos claramente vinculados al concepto de patrimonio, e identificar lo que dicen acerca de la evolución, por una parte, del concepto mismo de patrimonio en la ciudad de Quito y, por otra, de los actores involucrados en el campo patrimonial. Se trata de analizar los cambios en la gobernanza urbana de Quito desde su nombramiento por la Unesco en 1978, gobernanza aquí entendida como un espacio político que involucra no solo al gobierno municipal elegido, sino a una variedad de actores procedentes del sector público, privado o semipúblico (Le Galès, 1995). Se podrá ver cómo las políticas patrimoniales llevadas a cabo desde arriba por múltiples actores a diferentes niveles (local, nacional e internacional) han dado lugar a una cierta visión de la ciudad y de su patrimonio,
${ }^{1}$ Este deriva de una investigacion, apoyada por AGO$R A$ y por la Fundación PATRIMA (bttp://www. sciences-patrimoine.org), y cuyo objetivo es llevar a cabo un análisis comparativo de las politicas patrimoniales implementadas en varias ciudades de América Latina. territarias 32

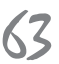


${ }^{2}$ El primer Plan Nacional del Buen Vivir fue presentado en 2008. Sus principios están fundados en la filosofía andina del Sumak Kawsay -nombre de la filosofia ancestral del pueblo kechwa que significa "Buen Vivir"-, y definidos en la Constitución de Montecristi en los Artículos 275 a 278 (2008): "el Buen Vivir requerirá que las personas, comunidades, pueblos y nacionalidades gocen efectivamente de sus derechos y ejerzan responsabilidades en el marco de la interculturalidad, del respeto a sus diversidades y de la convivencia armónica con la naturaleza” (art. 275). Uno de sus ejes es la "revolución cultural".

${ }^{3}$ El plano de propuesta elimina una parte de la cuadricula colonial, amplia las calles del núcleo fundacional destruyendo la arquitectura, aspecto que no se ejecutó.

\section{territarias 32}

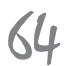

visión que ha ido evolucionando estos últimos años.

La hipótesis de esta investigación es que las políticas patrimoniales enfocadas en la rehabilitación del centro histórico de Quito han dejado durante tres décadas poco espacio para una visión más amplia y ciudadana del patrimonio. Sin embargo, esta situación ha evolucionado recientemente con la apertura de una nueva estructura de oportunidad política, según la expresión de Sydney Tarrow (1988), iniciada con la llegada al poder de Rafael Correa y el lanzamiento de la revolución ciudadana y del Plan Nacional del Buen Vivir. ${ }^{2}$ En este contexto, se verá en qué medida las tradicionales prácticas de gestión patrimonial han ido dejando espacio a unas políticas más integrales e inclusivas, que se podrían llamar políticas culturales.

\section{Cómo se construye el campo patrimonial en Quito}

Dos fechas se mencionan frecuentemente para identificar una evolución notable en la historia patrimonial de Quito: se trata de 1978, año del nombramiento del centro histórico como Patrimonio Cultural de la Humanidad, y de 1987, año del terremoto que produce el deterioro de una parte significativa de los edificios coloniales. No cabe duda de que el interés por el patrimonio colonial quiteño existía antes de esos acontecimientos, el Plan Regulador de Quito de 1942-1950, Jones de 1945 ya insistía en el valor arquitectónico del centro, y reconocía el patrimonio monumental como elementos aislados. ${ }^{3}$ Pero también es de notar que se produce un verdadero cambio en la gestión política y económica del tema patrimonial a partir de los años ochenta.

El concepto de campo de Pierre Bourdieu (2000) ayuda a analizar dicha evolución y entender cómo se crea un espacio bien definido, constituido por actores y procesos específicos, en el que el patrimonio se convierte en verdadero recurso político y económico para ciertas élites. $\mathrm{La}$ definición del campo político del sociólogo francés, que puede ser aplicada a cualquier otro sector del mundo social, permite entender las características del sector patrimonial que se constituye en Quito en las últimas décadas del siglo XX:

[...] el campo político [...] es un pequeño mundo social relativamente autónomo dentro del gran mundo social. Ahí encontramos varias propiedades, relaciones, acciones y procesos que se pueden encontrar en el mundo global, pero dichos procesos, dichos fenómenos tomarán aquí una forma peculiar. Es lo que nos dice la noción de autonomía: un campo es un microcosmo autónomo dentro del macrocosmo social [...] un mundo que obedece a sus leyes propias, que son distintas de las layes del mundo social ordinario (Bourdieu, 2000, p. 52).

La observación histórica de la emergencia de instituciones dedicadas a la gestión del patrimonio a partir del nombramiento de 1978, así como la de los actores que las manejan y de las acciones llevadas a cabo en el sector, conduce a hablar de 
la constitución de un verdadero campo patrimonial en Quito a partir de los años ochenta. Se crea así en Quito un grupo de actores especializados que, a pesar de ser distintos por su posición en el campo o su escala de intervención (local, nacional, internacional), tienen en común la legitimidad - otorgada por su presencia en el campo- para definir lo que es patrimonio o no lo es, y por lo tanto para diseñar las políticas o intervenciones en el sector del patrimonio. A partir de ahí, el patrimonio se convierte en un verdadero recurso político y económico para los diferentes actores del campo.

\section{Los actores legítimos del campo patrimonial}

¿Quiénes son los actores que constituyen el campo patrimonial en Quito a partir de los años ochenta? Se trata, como se ha mencionado, de actores procedentes de varios niveles de intervención. En la capital de Ecuador, como en la mayoría de las ciudades latinoamericanas, el nivel local y el nivel internacional han sido los más relevantes en las intervenciones patrimoniales. Este fenómeno, debido a la crisis económica que afecta a los países de la región en los años de transiciones democráticas, lleva a los Estados a implementar políticas económicas de ajuste estructural, frecuentemente asociadas con políticas de descentralización, y por lo tanto a un fuerte retiro del Estado de las problemáticas locales. Los municipios se convierten entonces en los mayores actores de la llamada gobernanza urbana, pero no son los únicos. La escasez de medios los obliga rápidamente a dejar espacio para otros actores procedentes de varios sectores. El concepto de gobernanza urbana, que introduce la idea de diversidad de actores, ayuda también aquí a "tomar en cuenta la capacidad estratégica de los actores, la diversidad de los procesos de legitimación, la dinámica de negociación entre actores" (Le Galès, 1995, p. 60).

En Quito, ambos niveles de intervención se desarrollan durante varios años de forma relativamente asociada y consensual. Por un lado, se crean instituciones a nivel local, principalmente a partir del sismo de 1987, que pone en evidencia la vulnerabilidad de los viejos edificios del centro histórico. El Fonsal (Fondo de Salvamento), que saca sus recursos de impuestos adicionales ${ }^{4}$ y de préstamos internacionales del Banco Interamericano de Desarrollo (BID), se convierte rápidamente en la institución más activa en las intervenciones de restauración del centro histórico, y de la ciudad en general. Con ella, trabajan otras instituciones procedentes mayoritariamente del sector privado o religioso, como por ejemplo la Fundación Caspicara, la Conferencia Episcopal, pero también el Colegio de Arquitectos o la Sociedad de Defensa del Patrimonio Artístico. Todos se comprometen en la lucha para la salvaguardia del patrimonio edificado a partir de finales de los años ochenta y una gran parte de ellos, a pesar de una evolución formal en algunos casos, permanecen en el campo patrimonial hasta la época actual. Es el caso por ejemplo del Fonsal, que se convierte en el Instituto
${ }^{4}$ El 6\% del impuesto a la renta recaudado en el Distrito Metropolitano. 
${ }_{5}^{5}$ Sitio internacional sobre la revitalización de centros históricos de ciudades de América Latina y del Caribehttp://www2.archi.fr/ SIRCHAL/

\section{territarias 32}

Metropolitano de Patrimonio (IMP) en 2011, con una vinculación más estrecha al municipio.

Esta colaboración institucional llama la atención de la comunidad internacional, cuya primera acción más visible en Quito es el préstamo del BID en 1995. Es la primera vez en la historia de esa institución que se otorga un préstamo para una intervención de rehabilitación urbana. La intervención de este actor en el campo patrimonial provoca un efecto dinamizador con la creación de nuevos gestores del patrimonio en el ámbito local, como la Empresa del Centro Histórico, empresa mixta dedicada a trabajar en asociación con el sector privado. A diferencia del Fonsal que no puede intervenir en los edificios de propiedad particular, la Empresa Centro Histórico está capacitada para fomentar el desarrollo económico del centro, asociando fondos privados y públicos a la vez que es un órgano ejecutor de proyectos municipales (Moreira, 2001).

La intervención del BID atrae la atención de otros sectores de la comunidad internacional, y más precisamente de la cooperación internacional en el centro histórico de Quito. En la década del noventa intervienen en Quito varios expertos internacionales, entre los cuales se destacan los actores de la cooperación española, como la AECI o la Junta de Andalucía, pero también los de la cooperación francesa con la ONG Pact-Arim, en materia de vivienda, o la red SIRCHAL, ${ }^{5}$ que escoge Quito como sede de su primer encuentro en octubre de 1998; y también Bélgica, mediante el proyecto Ecua-Bel y Polonia, para la restauración de edificios religiosos. Los grandes organismos internacionales intervienen también de forma directa como la Unesco, el Fondo de Patrimonio Mundial, el PNUD y ONU-Habitat que instala una sede permanente en la ciudad (Peyronniede Maxim, 2002).

De esta manera, no se puede olvidar el papel protagonista de personalidades extremadamente implicadas en la rehabilitación del patrimonio quiteño $y$, por lo tanto, en la rehabilitación del centro histórico. Alcaldes y personalidades del sector cultural local, nacional o internacional, han podido, por su posición en el campo patrimonial, asegurar la continuidad de políticas patrimoniales o la difusión de modelos de gestión entre Quito y el mundo.

Si se puede hablar de campo patrimonial, por la emergencia de varios actores especializados en el tema patrimonial, legitimados por su presentación oficial como expertos, también es de notar que, según Bourdieu (2000), un campo se caracteriza por la constitución de una autonomía respecto de otros campos, lo que le permite establecer leyes de funcionamiento propias. Esta autonomía la consiguen los actores patrimoniales en Quito con la creación de limitaciones no solo territoriales sino también jurídicas que les otorgan una gran libertad en la implementación de programas en el sector. Así, en el marco de la nueva división territorial administrativa para el Distrito Metropolitano de Quito en 1993, se crean varias administraciones zonales, entre las cuales está la Zona Centro Manuela Sáenz (1994), dentro de la cual se sitúa 
el centro histórico. A partir de entonces, los actores del campo patrimonial, expertos locales e internacionales en su mayor parte, pueden llevar a cabo de forma relativamente autónoma, durante varias décadas, las políticas de gestión patrimonial. Esta autonomía es lo suficientemente relevante para que Fernando Carrión y Manuel Dammert (2013) hablen de

la separación del centro histórico de Quito respecto de la ciudad, con lo cual, desde las políticas públicas $[\ldots]$ se niega la existencia de la ciudad y se intenta convertir al centro histórico en un enclave o una burbuja independiente de la misma (p. 192).

Se constituye así un espacio político que ha permitido durante años a las élites del campo patrimonial conservar el monopolio de la definición legítima del patrimonio.

\section{El patrimonio definido desde arriba}

Si uno de los mayores desafíos del campo político es, según Bourdieu (1996), la imposición de la visión legitima del mundo social, uno de los objetivos del campo patrimonial es la producción de la visión legítima del patrimonio, o lo que Eduardo Kingman (2004) llama también "una opinión autorizada sobre cultura, centros históricos, patrimonio" (p. 27). Y en Quito, así como en la mayor parte de las ciudades presentadas como ciudades patrimoniales, esta producción se hace principalmente mediante procesos de inventario y clasificación. En Quito, desde los comienzos de estos procesos, la definición va a ser mayoritariamente monumentalista, y limitada geográficamente a algunas zonas: el centro histórico y unos pocos barrios más identificados por su riqueza arquitectónica. ¿Cómo se impone esta visión? La constitución del campo patrimonial y la composición de sus élites pueden explicar por qué, hasta una época reciente, el patrimonio ha sido definido según esta visión dominante y excluyente.

Los primeros planos de urbanismo de los años cuarenta proponían una delimitación del centro histórico detallada que insistía en los monumentos religiosos y en las características arquitectónicas hispánicas. También, en los documentos preparatorios al nombramiento por la Unesco se destacan las iglesias, los conventos, las estatuas religiosas. Lo que está en coherencia con el texto de la convención de la Unesco de 1972 que define el patrimonio según criterios principalmente arquitectónicos. Los documentos de planificación municipal que se van a multiplicar a partir de los años noventa siguen esta misma visión y ponen el patrimonio edificado en el centro de los procesos de rehabilitación. Poco a poco, las definiciones legítimas del patrimonio muestran solo una parte de la ciudad de Quito, su centro histórico y otros tres barrios (también escogidos a principios de los años ochenta por su patrimonio edificado), Guápulo, Cotocallao y Chillogallo. El Plan Maestro de Rehabilitación de Áreas históricas 1989-1991 impone la visión monumen- territarias 32

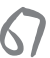


talista con base en unos ejes definidos por las plazas y los templos del centro histórico. El Plan especial de 2003 tampoco deja lugar a dudas respecto de la visión legítima del patrimonio: "el patrimonio edificado es la parte fundamental del valor tangible e intangible del centro histórico de Quito, por el cual la Unesco lo incluyó en la lista del Patrimonio Mundial" (MDMQ, 2003, p. 41).

Sin embargo, hay que matizar la visión que prevalece en la primera década del siglo XXI, ya que se introduce poco a poco una noción de desarrollo integral. El Plan Especial, por ejemplo, insiste en la necesidad de vincular cualquier intervención en el centro histórico con el resto de la ciudad. Se empieza a hablar de espacio público, vitalidad sostenible, articulación de los espacios urbanos, y ya no solo de elementos patrimoniales. Esta evolución se nota en los planes de desarrollo de la primera década del siglo XXI, planes ya no solo municipales sino metropolitanos, como por ejemplo el Plan Equinoccio 21 que insiste en la idea de desarrollo sostenible.

Se ve aquí que en esta década la visión monumentalista del patrimonio, sin dejar de ser dominante en el campo patrimonial, evoluciona hacia una visión más integrada y funcional que toma en cuenta las relaciones entre el centro histórico y el resto de la ciudad, o mejor dicho del distrito metropolitano, en aras de favorecer un desarrollo económico global.

Sin embargo, a pesar de estos cambios territarias 32 68 iniciados a principios del siglo XXI, los actores tradicionales del campo patrimonial siguen conservando el monopolio de la definición legítima en la que sigue prevaleciendo la visión monumentalista, justificada no solo por los criterios de la declaratoria de la Unesco sino también por la voluntad de atraer turismo y de diversificar las actividades económicas del centro histórico: si es de notar que el Fonsal interviene en los años noventa y en la primera década del siglo XXI en otros barrios fuera del centro, hay que subrayar que lo sigue haciendo en el patrimonio edificado, aspecto que atrae la mayor parte de las inversiones y también de la ayuda internacional. En resumidas cuentas, el campo patrimonial sigue hermético y poco flexible hasta principios del siglo XXI, tanto en su visión patrimonial como en la composición de sus actores. Estos últimos evolucionan poco, ya que los mismos expertos locales o internacionales siguen dominando el campo. Y aunque se lleven a cabo varios procesos participativos, se hacen exclusivamente para intervenir en el patrimonio edificado.

\section{5. ¿E1 Patrimonio para quién? Algunos efectos sociales en el centro histórico}

En Quito, a lo largo de las tres últimas décadas, las políticas patrimoniales, que se han llevado a cabo en el centro histórico, se han desarrollado paralelamente a un despoblamiento de esta misma zona. Las cifras son significativas: la población del centro pasa de 73225 habitantes en 1990 a 63785 habitantes en 2000, hasta llegar a 40870 en 2010 (MDMQ, 2013). 
El despoblamiento es evidente y plantea la cuestión de los efectos sociales de los programas de rehabilitación en el centro histórico. Quito se distingue aquí de otras ciudades donde los procesos de restauración han podido dar lugar a un proceso de gentrificación de los barrios, resultante de la inevitable especulación. Sin embargo, en Quito, las políticas de rehabilitación causan la salida de las categorías populares, sin que estas sean sustituidas por la población de clase media que el gobierno local quiere atraer para revitalizar la zona. El problema es tal que Pedro Jaramillo plantea en 2010 la cuestión de la sostenibilidad del centro histórico:

El atractivo para la vivienda pierde sustento debido a los altos precios de las propiedades lo que no viabiliza operaciones inmobiliarias rentables $[\ldots]$ esto nos lleva a concluir que en el caso de Quito, el mayor motivador para la preservación es el gobierno quien busca la promoción y recuperación del centro para flujos externos: turistas, eventos masivos $[\ldots]$ ( p. 4l).

Si es evidente que las políticas de restauración tratan de crear un nuevo dinamismo económico mediante las inversiones privadas, cuyo objetivo es incentivar la llegada masiva de turistas, la conclusión de Jaramillo nos lleva a preguntarnos en qué medida las políticas patrimoniales se dirigen también a los ciudadanos quiteños.

Estas políticas patrimoniales han influido fuertemente en la evolución demográfica de estos últimos treinta años en del centro histórico, dando lugar a lo que Eduardo Kingman (2004) identifica como una "administración de las poblaciones" (p. 30) por parte de unas élites que quieren ordenar la ciudad según criterios patrimoniales presentados como consensuales. Uno de los ejemplos más significativos de este ordenamiento de las poblaciones es el programa de reubicación de los comerciantes informales en el 2000 tras un largo periodo de negociación. Ya a partir de los noventa, la ocupación del espacio público por los ambulantes es presentada como un aspecto problemático en los diferentes documentos relativos al desarrollo urbano del centro histórico. Este mapa sacado del Plan Especial del CH de 2003 (p. 11) identifica claramente las zonas de comercio ambulante como zonas problemáticas (ver figura 1).

La repetición de la expresión recuperación del espacio público, a partir de entonces, en los discursos oficiales es significativa de la visión dominante de las élites del campo patrimonial. Se trata efectivamente de legitimar una medida económica (erradicación del comercio ambulante), gracias a un discurso patrimonial dominante y presentado como consensual. Se puede hablar aquí de violencia simbólica, según la expresión de Pierre Bourdieu (1996), ya que el discurso produce unos efectos sociales notables mediante la identificación de actividades ilegales y, por lo tanto, de ciudadanos no deseados en el espacio público. Estos procesos son significativos de una verdadera lucha de poder que enfrentan las élites del patrimonio y una parte territarias 32 


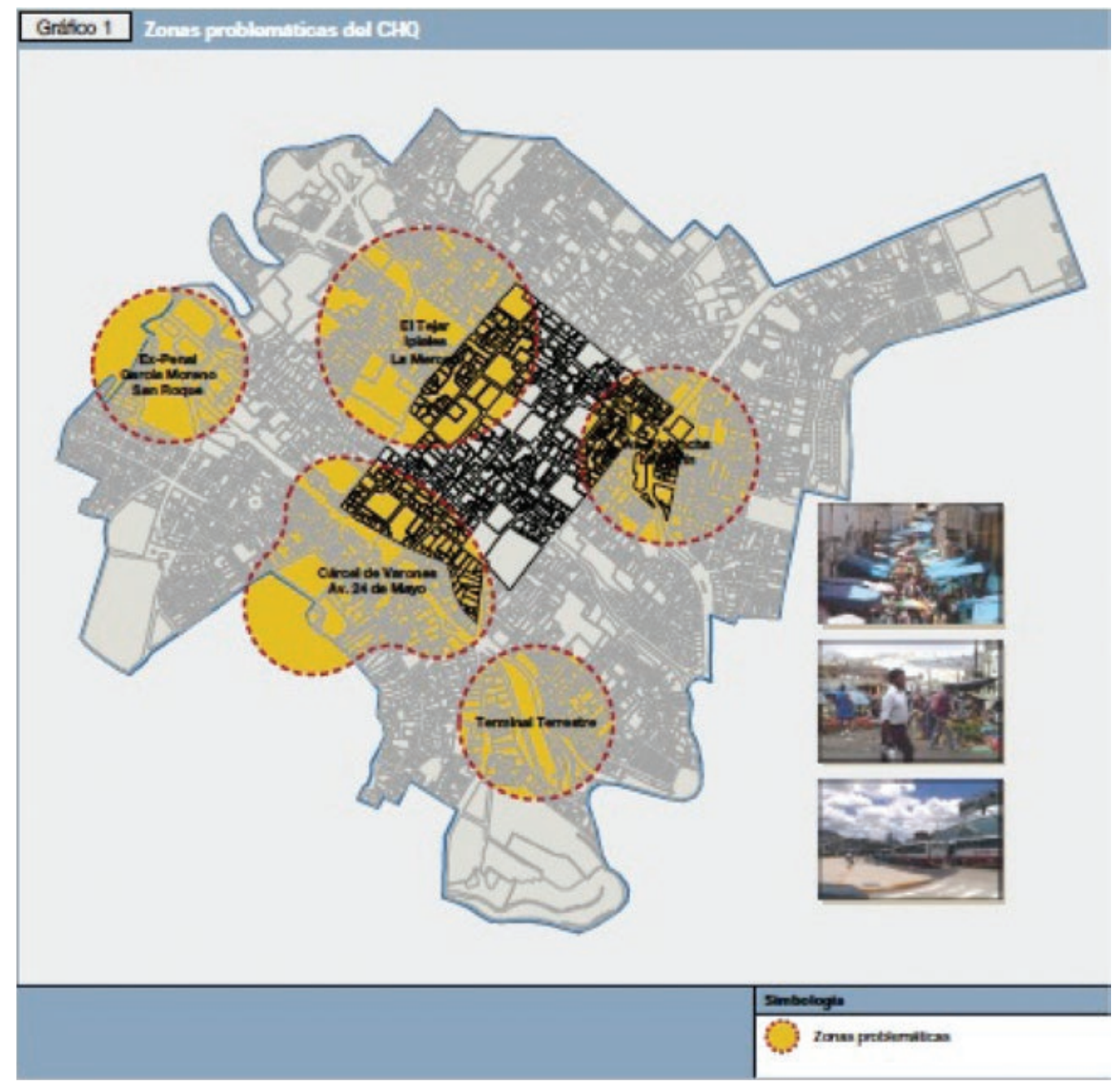

Fuente: Plan Especial del Centro histórico, MDQM, 2003, p.11

de los ciudadanos de Quito. El trabajo de investigación de Lucía Durán (2014) en un barrio emblemático del centro, La Ronda, es revelador de la existencia de un conflicto

territarias 32 70 que no emerge como conflicto visible en el espacio público por la fuerza misma del discurso patrimonial. Durán (2014) analiza los múltiples mecanismos que permiten a las élites justificar y legitimar un proceso de restauración del barrio y de presentarlo 
como consensual, a pesar de las oposiciones locales de los vecinos. Mediante procesos de estigmatización de la zona afectada (presentación de La Ronda como una zona roja), se construye poco a poco una visión dominante de "lo digno de conservar", "lo digno de recordar", y, por lo tanto, de "indignidad" (p. 149).

Y si no se puede negar que los procesos de intervención patrimonial se han desarrollado en Quito de forma más consensual que en otras ciudades suramericanas (los comerciantes fueron reubicados en centros comerciales de la zona tras un proceso de negociación que duró varios años), hay que admitir también que los efectos sociales en términos demográficos han sido catastróficos. El despoblamiento del centro histórico a partir de los noventa hace eco a la salida de las élites de esta misma zona desde los años setenta, época de bonanza petrolera durante la cual las categorías sociales más favorecidas salieron del centro para instalarse en el norte de la ciudad. Las políticas de recuperación del centro histórico, llamadas más recientemente políticas de revitalización, no han conseguido frenar el fenómeno de despoblamiento a lo largo de la segunda mitad del siglo XX. El centro histórico no logra atraer nuevas categorías de población, ni siquiera nuevas inversiones turísticas. Además, la privatización de varios espacios patrimoniales (comercios, restaurantes, hoteles de lujo) se añade a este fenómeno de despoblamiento, lo que ha llevado a varios observadores a cuestionar la centralidad del centro histórico como espacio público, e incluso a hablar de "un abandono físico y simbólico del centro histórico" (Carrión \& Dammert, 2013, p. 190).

\section{La cultura local en un contexto nacional reformista}

En 2009, Augusto Barrera es elegido alcalde de Quito. Este hombre, cercano a la línea ideológica del presidente Rafael Correa, va a implementar unos programas bastante innovadores en el sector del patrimonio, y de la cultura en general. Mientras que un alcalde como Paco Moncayo trataba de posicionar la ciudad de Quito en la primera década del siglo XXI como nodo territorial y regional (el proyecto del nuevo aeropuerto internacional diseñado durante su mandato de Quito era emblemático de esa visión estratégica), Augusto Barrera le da la prioridad a una visión más inclusiva de la ciudad, al insistir en las problemáticas sociales internas. Una de las prioridades oficiales es el establecimiento de una política cultural más democrática, lo que supone también una definición oficial de la cultura mucho más amplia que la que prevalecía en los programas de planificación anteriores:

Entendemos por cultura todo lo que el ser humano ha creado a lo largo de la historia: el mundo de la vida y el mundo de las cosas [...] Son cultura los mitos, las artes, las esculturas, las ciencias, las formas religiosas, y también los modos de cocinar, de construir casas, las modas y vestimentas, la manera de divertirse, de transitar y de hacer fiesta, de escribir, de investigar (MDMQ, 2012, p. 87). territarias 32 
Es interesante notar que las referencias al Patrimonio en los documentos oficiales evolucionan hacia una visión también más plural y que se superponen con las definiciones de la cultura. Emerge así poco a poco la visión de un patrimonio que puede ser también inmaterial o intangible, lo que supone un cambio radical con las visiones anteriores:

[...] el patrimonio intangible es vasto en la medida que se refiere a todas las costumbres, tradiciones, manifestaciones culturales, quehaceres y normas propias de la diversidad cultural existente en el Distrito, y que han sido apropiados, desarrollados, mantenidos, transformados por diferentes grupos culturales (MDMQ, 2012, p. 87).

¿A qué se debe esta evolución en el discurso oficial? En realidad se trata menos de una evolución del campo patrimonial (que como se verá sigue gestionado por instituciones tradicionales), que el reflejo de una nueva orientación política nacional. Las evoluciones en el discurso oficial del gobierno de Quito no pueden entenderse sin una referencia al contexto nacional, y más precisamente a las orientaciones ideológicas del gobierno de Rafael Correa. Una de las características mayores de la revolución ciudadana, impulsada por este presidente consiste en presentar los derechos culturales como derechos fundamentales. La nueva Constitución de 2008 los define como elementos fundamentales del nuevo régimen de Buen Vivir (Artículos

\section{territarias 32} 21 a 25 de la Constitución), y proclama una definición del patrimonio que rompe radicalmente con la visión tradicional: elementos como las lenguas, las tradiciones orales, las creaciones artísticas, científicas, tecnológicas, son considerados como patrimonio tangible e intangible, y ocupan por primera vez un lugar destacado al lado de los tradicionales elementos patrimoniales tales como edificaciones, monumentos, $\mathrm{y}$ colecciones (Artículo 379).

Los Planes Nacionales del Buen Vivir (2009-2013 y 2013-2017) inspirados en los principios definidos por la Constitución otorgan un papel destacado a la Revolución Cultural:

La Revolución Cultural, que genera nuevos conceptos y valores ciudadanos para constituir una sociedad crítica, emprendedora, creativa y solidaria. Se vuelven fundamentales las industrias culturales y la democratización de la palabra. También implica una revolución educativa para formar ciudadanos con otra mentalidad, con valores asentados, con ética y con autoestima. El Buen Vivir significa, sobre todo, tener en el país una población con una gran dosis de autoestima y de confianza colectiva" (Plan Nacional del Buen Vivir, 2013-2017, p. 18).

En el marco de este nuevo contexto nacional, en el que emergen también nuevas instituciones como el Ministerio de la Cultura en 2007, se pueden entender mejor los proyectos locales del gobierno de Quito a partir de 2009, en el sector patrimonial. La estrategia es trabajar a nivel metropolitano a partir de un diagnóstico que 
demuestra la gran desigualdad del reparto territorial de las instituciones culturales. Así, se crean 44 centros de desarrollo comunitario (cinco en el centro histórico) que proporcionan diversos servicios (ayuda escolar, talleres artísticos, actividades para ancianos, formación informática, etc.) y se inicia el lanzamiento de una cartografía del patrimonio inmaterial de Quito. La línea ideológica está claramente definida: se trata de democratizar el acceso a la cultura y al patrimonio.

\section{Renovar la idea de patrimonio: el ejemplo de Cuéntame Tu Quito}

¿Cómo se traduce concretamente la vinculación entre cultura y patrimonio? El estudio de la campaña municipal Cuéntame tu Quito permite analizar esta evolución que, sin romper con el marco establecido por el campo patrimonial tradicional, abre puertas para establecer una nueva visión de la relación entre el ciudadano y su ciudad. Organizada en 2013 para celebrar los 35 años del nombramiento de la Unesco, esta campaña se da como objetivo la consulta a los ciudadanos quiteños acerca de lo que ellos consideran lo que es o no es patrimonio de la ciudad. Las preguntas son las siguientes: “¿Qué es patrimonio? ¿Cómo podemos saber qué es parte de nuestra memoria colectiva y qué no? ¿Cuáles son nuestros saberes? ¿Qué nos enorgullece?”.

Más allá de la reflexión pública acerca del concepto mismo de patrimonio que esta campaña promueve, se pueden destacar varios elementos que demuestran una evolución notable, respecto de la situación anterior que prevalecía en el campo patrimonial. En primer lugar, la campaña se basa en un amplio proceso participativo que le permite al gobierno municipal no solo legitimar una nueva visión patrimonial sino también promover la política cultural llevada a cabo desde la elección de Augusto Barrera en 2009, en un momento clave que es el de la preparación de su reelección. La promoción de la política cultural se hace mediante el uso de 16 de los 44 centros de desarrollo comunitario del distrito metropolitano de Quito para llevar a cabo varios talleres participativos en los que los ciudadanos reflexionan acerca del concepto de patrimonio, de distintos modos (radio, escritos, dibujos, fotos). Otras consultas se hacen también en el espacio público (escuelas, plazas, parques), lo que permite dar una gran visibilidad al proceso. Asimismo, la campaña utiliza las redes sociales para ampliar su base participativa, y crea un portal interactivo y una cuenta Facebook.

En segundo lugar, la campaña Cuéntame tu Quito muestra otra forma de gestionar el tema del patrimonio a nivel local. Los dos actores que lideran la campaña son el Instituto Metropolitano del Patrimonio (IMP) y la Fundación Museos de la Ciudad (FMC), y si el primero es un actor tradicional del campo patrimonial de Quito (ex Fonsal), el segundo es relativamente nuevo. La Fundación Museos de la Ciudad es creada en 2006 para coordinar las mayores instituciones culturales de la ciudad. Tras el cambio de administración en 2009 , cambia de estructura interna e territarias 32 
instaura un organigrama más horizontal, reflejo de la voluntad democratizadora en el sector cultural. El discurso de los líderes de ambas instituciones deja ver el cambio de estrategia oficial en la gestión del tema patrimonial:

- La idea era actualizarnos, salir de nuestras oficinas, y ¡lo conseguimos! Claro que nos vamos a dejar de restaurar iglesias, casas coloniales. Pero lo que resulto de la consulta, es que van creciendo otras ideas del patrimonio, $\mathrm{CO}^{-}$ mo el patrimonio natural. Por eso, este año, intervenimos en el Panecillo y las Quebradas (Armijos, comunicación personal, IMP, febrero 17, 2014).

- Hay una idea demasiado unesquiana del patrimonio, y por lo tanto una brecha con los ciudadanos, intentamos ahora mirar la ciudad desde abajo (Rodríguez, FMC, comunicación personal, febrero 19, 2014).

Se nota aquí el tono claramente reformista de un discurso que insiste en la democratización de la cultura, y en la voluntad de romper con las políticas anteriores presentadas como demasiado elitistas. Los resultados de la campaña presentan efectivamente una idea renovada del $\mathrm{Pa}-$ trimonio. Expuestos en el nuevo parque urbano Qmandá inaugurado en enero de 2014 , bajo distintas formas (exposición de fotos, cajas gigantes, textos), los resultados de la campaña Cuéntame tu Quito muestran otra ciudad, lejos de la tradicional imagen colonial promovida por los actores patrimoniales. La exposición muestra los parques, los jardines, los paisajes de la ciudad, el patrimonio natural de Quito. Pero también expone fotos de la gente de Quito, sus comerciantes, sus habitantes, sus mercados, sus niños jugando en las calles. Al lado de algunas fotos de monumentos e iglesias se destacan otras formas de patrimonio, más cotidiano y más cercano a los ciudadanos. Las cajas presentan también las formas de patrimonio intangible que son las fiestas, los colores, los saberes y tradiciones que hacen la identidad de los quiteños. De esta manera, la exposición muestra un patrimonio que no se reduce al centro histórico, e intenta presentar la ciudad en su globalidad.

Estamos aquí lejos de la visión monumentalista que prevalecía en las décadas anteriores. Sin embargo, hay que matizar el significado de la campaña en la evolución del campo patrimonial: si se autorizan aquí otras formas de expresiones patrimoniales, no dejan de ser visiones organizadas y promovidas desde arriba por los actores institucionales de la cultura. Tampoco hay que olvidar los objetivos electoralistas de un proceso organizado en vísperas de las elecciones municipales de mayo de 2014.

No obstante, las nuevas orientaciones políticas en Quito desde 2009 han dado lugar a la creación de nuevos espacios en los que los ciudadanos pueden expresar una forma renovada de expresión cultural. Uno de los ejemplos más significativos de estos procesos es el Parque urbano Qmandá.

\section{territarias 32}




\section{El Qmandá: ‘̨creando patrimonio desde abajo?}

Si fue el escenario privilegiado de los resultados la campaña Cuéntame tu Quito, el Qmandá es ante todo el primer parque urbano de Ecuador en reunir las actividades deportivas y las culturales.

Situada al límite sur del Centro histórico de Quito, en el barrio de la Loma, esta obra forma parte de los proyectos municipales de revitalización del centro histórico. Se inicia en 2013 y es inaugurada en enero de 2014. El parque urbano, que consta de $15000 \mathrm{~m}^{2}$ de construcción en un terreno de $35000 \mathrm{~m}^{2}$, ofrece a los habitantes de Quito varias infraestructuras de deporte y de ocio, a precios bajos: siete piscinas, gimnasios, un auditorio, canchas deportivas, biblioteca, salas de exposición. Además, la construcción obedece a normas de protección medioambientales y utiliza energías renovables (ver figuras 2 y 3 ).

Figura 2. El parque urbano Qmandá

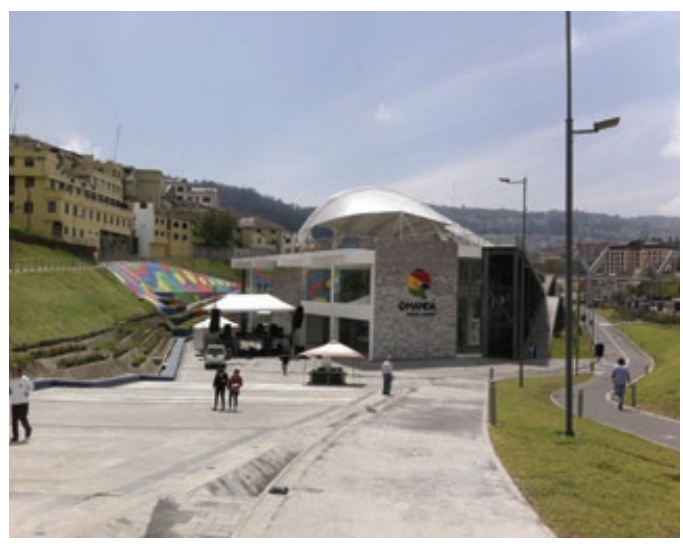

Fuente: elaboración propia.
Figura 3. El parque urbano Qmandá

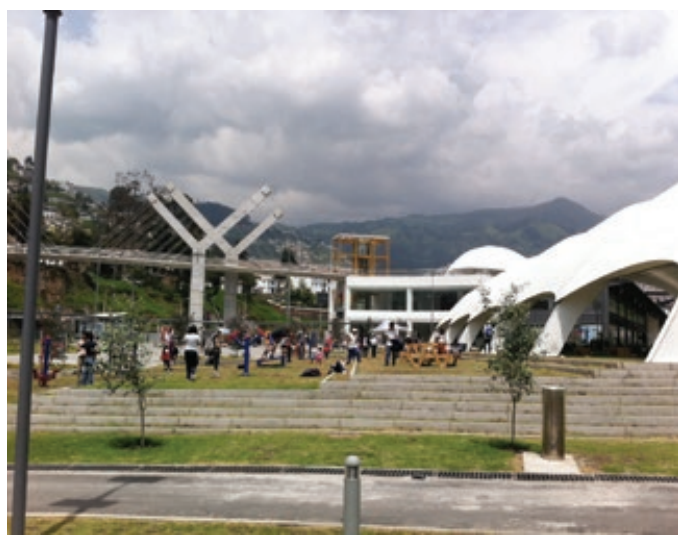

Fuente: elaboración propia.

El Parque urbano Qmandá ofrece a cualquier observador de la historia urbana un terreno rico de enseñanzas para entender la evolución de la gestión cultural en Quito y, más allá, los cambios en la relación entre el quiteño y el patrimonio de su ciudad. Se puede, en efecto, llevar el análisis de la relación entre el Qmandá y el concepto de patrimonio según dos perspectivas: su valor simbólico y su valor de uso. El valor simbólico del parque urbano reside principalmente en la imagen de puente que ofrece en la ciudad, y eso a varios niveles. Puente espacial, en primer lugar, ya que representa una relación entre el Centro histórico y el resto de la ciudad por su posición geográfica. Puente social, ya que se dirige mayoritariamente a categorías sociales populares. Puente histórico, entre un pasado colonial (al límite del centro histórico) y el futuro representado por la modernidad de las infraestructuras. Pero, a un nivel más concreto, el Qmandá representa ante todo 
una nueva manera de vivir el patrimonio que se manifiesta en el uso mismo que los quiteños hacen de esa obra.

Las primeras cifras oficiales del uso concreto del parque urbano son comunicadas en mayo de 2014. Tras las elecciones municipales en las que gana el candidato de derechas, la Fundación Museos, que gestiona el parque desde su inauguración, presenta públicamente su informe de gestión. Las cifras son significativas del gran éxito de esta nueva infraestructura: más de un millón de personas han visitado el parque urbano entre enero y mayo de 2014 , casi todos quiteños. En unos pocos meses, el Qmandá se ha convertido en la estructura cultural más utilizada de la capital, como lo demuestra la tabla 1 (FMC, 2014, p. 29).

El éxito del Qmandá respecto de las otras instituciones se debe matizar, ya que se trata no solo de una estructura cultural sino también de un centro de ocio y de de- porte. Sin embargo, el alto número de visitantes en un periodo tan reducido conduce a preguntarse en qué medida se puede analizar el fenómeno como una nueva relación del habitante de Quito con su patrimonio. El uso cotidiano que los habitantes hacen del parque urbano, tanto a nivel deportivo como cultural, permite pensar que emerge aquí una nueva forma de experimentar la cultura, volviendo al primer sentido de la palabra patrimonio entendido como pertenencia. Mucho más que en otras estructuras culturales o patrimoniales de la ciudad, el quiteño puede apoderarse del lugar. En ese sentido, el uso del Qmandá por los habitantes de la ciudad puede permitir la emergencia de una cultura urbana, tal como la define el filósofo francés Olivier Mongin (2005): "volver a encontrar lo local, volver a encontrar el sentido del lugar, volver a encontrar el sentido de la experiencia urbana en un lugar" (p. 259). En

Tabla 1. Registro de personas beneficiadas en actividades museos de 2009 a 2014

\begin{tabular}{|r|c|r|r|r|r|r|}
\hline $\begin{array}{c}\text { Museo de } \\
\text { la ciudad }\end{array}$ & $\begin{array}{c}\text { Yaku Parque } \\
\text { Museo del } \\
\text { Agua }\end{array}$ & $\begin{array}{c}\text { Museo } \\
\text { Intectivo } \\
\text { de Ciencia }\end{array}$ & $\begin{array}{c}\text { Centro de Arte } \\
\text { Contemporáneo }\end{array}$ & $\begin{array}{c}\text { Museo del } \\
\text { Carmen } \\
\text { Alto }\end{array}$ & $\begin{array}{c}\text { Qmandá } \\
\text { Parque } \\
\text { Urbano }\end{array}$ & Total \\
\hline 57457 & 130899 & 63357 & - & - & - & 251713 \\
\hline 63335 & 142094 & 75214 & - & - & - & 280643 \\
\hline 102266 & 153307 & 91236 & 52281 & - & - & 399090 \\
\hline 75106 & 135205 & 87379 & 76891 & - & - & 64581 \\
\hline 120321 & 168779 & 228349 & 115346 & 13750 & - & - \\
\hline $\mathbf{4 4 6 8 0 6}$ & $\mathbf{8 0 0 1 9 8}$ & $\mathbf{5 8 3 4 6 3}$ & $\mathbf{2 7 5 6 1 5}$ & $\mathbf{2 4 6 9 4}$ & $\mathbf{1 0 5 3 7 8 5}$ & $\mathbf{3 1 8 4 5 6 1}$ \\
\hline
\end{tabular}

Fuente: Informe de gestion, Fundación Museos de la Ciudad, 2014, p. 29. 
ese sentido, el Parque Qmandá pasa a ser patrimonio de los quiteños, un patrimonio creado y renovado de forma cotidiana por los propios ciudadanos. Representa pues una forma de hacer patrimonio, con una visión renovada que no solo se aleja de las definiciones tradicionales del patrimonio, sino también del uso que permite. Aquí, el patrimonio es también cultura ciudadana y cultura viva, como obra asequible a los habitantes, que a su vez también puedan actuar en ella.

En resumidas cuentas, la política cultural democratizadora llevada a cabo desde 2009 alcanza aquí, más que en cualquier otro lugar de la ciudad, sus objetivos de llegar hasta la gente y de permitir que el espacio patrimonial ya no sea visto como un lugar de conflicto, de poder o de exclusión, sino como un lugar de reconciliación.

\section{Conclusiones}

El análisis de las políticas implementadas estos últimos años por el gobierno municipal demuestran una voluntad clara de abrir el concepto de patrimonio y de vincularlo estrechamente con el de cultura. Esta línea política local entra lógicamente en coherencia con el marco ideológico e institucional nacional, ya que se crea en 2013 un Ministerio de la Cultura y del Patrimonio que viene a sustituir el primer Ministerio de la Cultura creado en 2007.

Los proyectos de democratización cultural llevados a cabo en Quito pueden, por lo tanto, abrir el camino hacia una reflexión nacional acerca de la definición misma de patrimonio. Esta reflexión se vuelve hoy imprescindible en Quito para fortalecer la relación entre el habitante y su entorno, en un contexto urbano ya muy lejos de la imagen de ciudad hispánica promovida por los actores patrimoniales tradiciones: Quito se ha convertido en una ciudad de migración que recibe cada año 275000 personas -es decir un séptimo de la población total del distrito metropolitano- entre las cuales se destacan nacionalidades muy diversas. Frente a la evolución demográfica de la ciudad, se plantea de forma más urgente la necesidad de definir lo que puede seguir siendo patrimonio de los habitantes de Quito, pero también lo que puede convertirse en un nuevo patrimonio, según una definición renovada.

En este sentido, sería interesante analizar a largo plazo en qué medida iniciativas como Cuéntame tu Quito pueden implantarse a largo plazo, más allá de un periodo electoral, y convertirse así en una verdadera herramienta de la ciudadanía, fortaleciendo la relación entre el ciudadano y su ciudad. El Qmandá puede ser también un modelo en este proceso y abrir caminos para una renovación del concepto de patrimonio en el marco de una nueva cultura urbana, que, según Olivier Mongin (2005) "no puede ser exclusivamente patrimonial, artística, arquitectónica, sino que exige que un espacio tome una forma política y que encuentre una coherencia para evitar el estallido urbano" (p. 17). Solo de este modo podrán los habitantes de Quito ejercer un derecho a la ciudad que también se puede, sencillamente, llamar ciudadanía. territarias 32

77 


\section{Referencias}

Bourdieu, P. (2000). Propos sur le champ politique. Lyon: Presses universitaires de Lyon.

Bourdieu, P. (1996). Champ politique, champ dessciences sociales, champ journalistique. Lyon: Ed. Cahiers de recherche, 15.

Carrión, F., \& Dammert, M. (2013). Centro histórico de Quito: ¿patrimonio de la humanidad o del mercado? En M. Fiori (Ed.), Revivir el centro histórico, Barcelona, la Habana, ciudad de México y Quito (pp. 181-214). Barcelona: Ed. UOC.

Cifuentes, C. (2008). Planning the Patrimonial Areas of Quito. Revista de la Organización Latinoamericana y del Caribe de Centros Históricos, 1, 101-114.

Durán, L. (2014). Patrimonio cultural, políticas de representación y estigma : una mirada desde el Centro Histórico de Quito. En Grimson (Comp.), Culturas politicas y politicas culturales (pp.145162). Buenos Aires: Ed. Böll Cono Sur. Estado de la República de Ecuador (2008). Constitución de la República del Ecuador. Montecristi: Asamblea Constituyente.

Fundación Museos de la Ciudad (2014). Informe de gestión 2009-2014. Quito: Ed. FMC.

Jaramillo, P. (agosto, 2010). The Sustainability of Urban Heritage Preservation: the case of Quito. Discussion paper, InterAmerican Development Bank.

tersitarias 32 78
Kingman, E. (2004). Patrimonio, políticas de la memoria e institucionalización de la cultura. Iconos, 20, 26-34.
Le Galès, P. (1995). Du gouvernement des villes à la gouvernance urbaine. Revue Française de Science Politique, 45(1), 57-95.

Moreira, O. M. (2001). El Centro Histórico de Quito: un modelo mixto de gestión. En F. Carrión (Comp.), Centros Históricos de América Latina y del Caribe (pp. 253-273). Quito: FLACSO, sede Ecuador Ed.

Municipio del distrito metropolitano de Quito-Instituto Metropolitano de Patrimonio (2013). Plan de gestión integral del centro histórico de Quito, Quito: Ed. MDMQ

Municipio del distrito metropolitano de Quito (2012). Plan metropolitano de desarrollo 2012-2022, Quito: Ed. MDMQ

Municipio del distrito metropolitano de Quito (2004). Plan Equinoccio 21, Quito: Ed. MDMQ.

Municipio del distrito metropolitano de Quito-Junta de Andalucía (2003). Centro histórico de Quito: Plan Especial, Quito: Ed. MDMQ.

Municipio de Quito-Agencia Española de Cooperación Internacional (1989). Plan Maestro de rehabilitación de áreas históricas 1989-1991, Quito: Ed. MDMQ.

Municipio de Quito (1945). Memoria descriptiva del proyecto del plan regulador para la ciudad de Quito, (proyectista Jones-Odriozola). Quito: Ed. MDMQ. Mongin, O. (2005). La condition urbaine. Paris: Seuil. 
Peyronnie, K., \& De Maximy R. (2002). Quito inesperado: de la memoria a la mirada crítica. Quito: Abya-Yala, IFEA.

Secretaría Nacional de Planificación y Desarrollo-Senplades (2013). Plan Nacional del Buen Vivir, 2013-2017, Quito: Ed. República del Ecuador.
Tarrow, S. (1988). National Politics and Collective Action. Annual Review of Sociology, 14, 421-440.

Unesco, (noviembre de 1972). Convention Concerning the Protection of the World Cultural and Natural Heritage. Paris: Ed. Unesco. 
